\title{
The Unwound Cochlea: A Specific Imaging Marker of Branchio-Oto-Renal Syndrome
}

\author{
(D) A. Hsu, (D) N. Desai, and (D) M.J. Paldino
}

\begin{abstract}
BACKGROUND AND PURPOSE: Branchio-oto-renal syndrome is an important syndromic cause of hearing loss. Our aim was to determine the test characteristics of the unwound cochlea on temporal bone CT for the diagnosis of branchio-oto-renal syndrome in a cohort of children with hearing loss.
\end{abstract}

\begin{abstract}
MATERIALS AND METHODS: Patients were identified retrospectively with a clinical diagnosis of branchio-oto-renal syndrome and CT imaging of the temporal bones. Age-matched controls were also identified with sensorineural hearing loss not related to a diagnosis of branchio-oto-renal syndrome and CT imaging of the temporal bones. All examinations were reviewed by 2 neuroradiologists blinded to the diagnosis of branchio-oto-renal syndrome versus controls for the absence/presence of an unwound cochlea defined as anteromedial rotation and displacement of the middle and apical turns away from the basal turn.
\end{abstract}

RESULTS: The final study group comprised 9 patients with branchio-oto-renal syndrome (age range, 1-14 years; mean age, $8.0 \pm 4.3$ years) and 50 control patients (age range, 1-16 years; mean age, $7.9 \pm 4.1$ years). The cochlea was subjectively abnormal in all 9 patients. In 8 patients (89\%), imaging demonstrated a typical unwound cochlear morphology. By contrast, none of the control subjects demonstrated an unwound cochlea on either side. Statistically, the unwound cochlea was significantly more frequent in the branchio-oto-renal group compared with controls $(P<.001)$. The unwound cochlea was $89 \%$ sensitive and $100 \%$ specific for the diagnosis of branchio-oto-renal syndrome.

CONCLUSIONS: The unwound cochlea is a specific imaging marker of branchio-oto-renal syndrome. These findings further support the diagnostic accuracy and therefore the utility of temporal bone imaging in the diagnosis of this disorder.

ABBREVIATION: $B O R=$ branchio-oto-renal syndrome

B ranchio-oto-renal syndrome (BOR) is a rare autosomal dominant disorder that manifests as hearing loss, branchial fistulas, malformations of the ear, and renal anomalies. ${ }^{1}$ It is one of the most common syndromic causes of hearing loss, with an estimated prevalence of $1: 40,000 .^{2-4}$ In the absence of family history, the diagnosis of BOR is made on the basis of the identification of 3 major criteria (second branchial arch anomalies, hearing loss, preauricular pits, auricular malformation, and renal anomalies) or 2 major and 2 minor criteria (preauricular tags or anomalies of the external auditory canal, middle ear, or inner ear). Expression of these findings is variable, however, resulting in the potential for a delayed or missed diagnosis, which can adversely impact the language and social development of these children. ${ }^{2,5-7}$ In addi-

Received July 2, 2018; accepted after revision September 4

From the Department of Radiology, Texas Children's Hospital, Houston, Texas.

Please address correspondence to Michael J. Paldino, MD, Texas Children's Hospital, 6701 Fannin St, Houston, TX 77030; e-mail: mjpaldin@texaschildrens.org

http://dx.doi.org/10.3174/ajnr.A5856 tion, though frequently silent at presentation, renal anomalies are a substantial cause of morbidity during the life span of these individuals. ${ }^{8}$ An early and definitive diagnosis prompting a search for associated anomalies would therefore be of great value in the management of these patients. In this regard, genetic testing for mutations in the EYA1, SIX1, and SIX5 genes represents a promising adjunct. ${ }^{2,7,9}$ Currently, however, only approximately $50 \%$ of affected individuals have detectable gene mutations. ${ }^{2,7,10}$ Furthermore, an index of suspicion is required to justify the cost of such analyses, especially in light of the rarity of this disorder among all patients presenting with abnormal hearing. Given that most patients with BOR manifest hearing loss, temporal bone imaging represents an appealing opportunity to make the diagnosis. ${ }^{7,11,12}$ Unfortunately previous reports are heterogeneous, and the specificity of imaging findings for this syndrome is only rarely reported. ${ }^{10}$ As a result, temporal bone anomalies currently constitute minor criteria for the diagnosis. ${ }^{8}$ Robson $^{3}$ reported that a distinctive "unwound appearance" of the cochlea, characterized 
by an anterior offset of the hypoplastic middle and apical turns away from a tapered basal turn, is characteristic of BOR. The diagnostic accuracy of this particular cochlear finding, however, is yet to be assessed. The goal of this study, therefore, was to determine the test characteristics of the unwound cochlea on temporal bone CT for the diagnosis of branchio-oto-renal syndrome in a cohort of children with hearing loss.

\section{MATERIALS AND METHODS \\ Subjects}

This Health Insurance Portability and Accountability Act-compliant study was approved by the local institutional review board. Informed consent was waived. This article conforms to Standards for Reporting of Diagnostic Accuracy Studies guidelines for reporting diagnostic accuracy. ${ }^{13}$ Consecutive patients were identified retrospectively from a search of existing patient data at a single institution. Inclusion in this study was based on the following criteria: 1) a diagnosis of BOR based on the reference standard: clinical evaluation by a member of the department of otorhinolaryngology according to standard criteria ${ }^{8}$; and 2) available CT imaging of the temporal bones. Control patients were identified with the following inclusion criteria: 1) sensorineural hearing loss (documented by a formal audiologic evaluation) deemed not related to a diagnosis of BOR after evaluation by an otorhinolaryngologist, and 2) available CT imaging of the temporal bones.

\section{Imaging}

Representative CT imaging protocol was the following: volumetric acquisition from above the ear to below the base of skull; 120 $\mathrm{kV}$ (peak); $220 \mathrm{~mA}$ (0-11 years of age) or $230 \mathrm{~mA}$ (12-18 years of age). Reconstructions were the following: 1-mm-slice axial and coronal reformats in a standard FOV $(22 \mathrm{~cm})$ and $0.5-\mathrm{mm}$-slice bilateral temporal bone axial and coronal reformats in FOV of $9.6 \mathrm{~cm}$.

\section{Image Review and Analysis}

All imaging examinations were reviewed by 2 neuroradiologists, each with approximately 10 years of subspecialty experience in pediatric neuroimaging. Review was performed blinded to the diagnosis of BOR versus controls. The primary measure in this study was a subjective assessment of the absence/presence of an unwound cochlea. In particular, the unwound cochlear dysmorphology consists primarily of anteromedial angulation and displacement of the middle and apical turns of the cochlea away from the basal turn (Fig 1). In addition, the basal-middle turn interval was measured as the maximal distance between the basal (any part) and middle (any part) turns of the cochlea on axial CT images of the temporal bone. This measurement was performed in an attempt to quantify what we believe to be the fundamental dysmorphology of the unwound cochlea and, thereby, corroborate the subjective assessment. Finally, the presence of any other cochlear and inner ear abnormalities was assessed and tabulated.

The frequency of the unwound cochlea in patients with BOR was compared with that in control subjects using the Wilcoxon rank sum test. The sensitivity, specificity, and positive predictive value of an unwound cochlea for the diagnosis of BOR were calculated in a standard fashion. Agreement between readers was
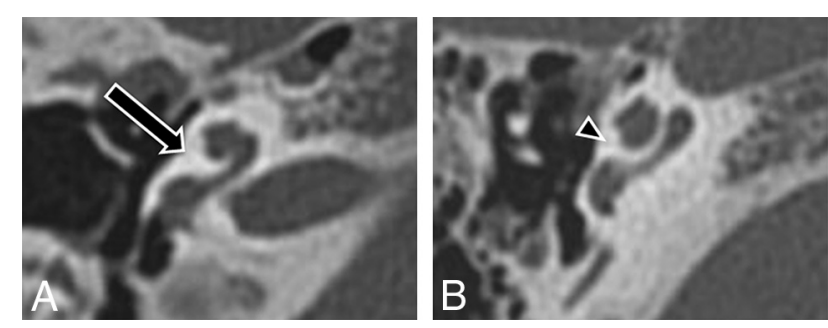

FIG 1. Unwound cochlea. A, Axial CT image through the right cochlea of a patient with BOR demonstrates characteristic unwound dysmorphology with anteromedial rotation and displacement of the middle turn away from a tapered basal turn (arrow). B, Axial CT image through a normal right cochlea demonstrates normal apposition of the middle and basal turns (arrowhead).

measured using the Cohen $\kappa$. Inconsistencies were settled by consensus re-review.

\section{RESULTS}

\section{Patients}

The final study group comprised 9 patients with a diagnosis of branchio-oto-renal syndrome (age range, 1-14 years; mean age, $8.0 \pm 4.3$ years; 6 boys, 3 girls). All patients underwent CT imaging from July 2013 through May 2017. All patients with BOR had hearing loss at a formal audiologic evaluation. The control group consisted of 50 patients (age range, 1-16 years; mean age, $7.9 \pm$ 4.1 years; 26 boys, 24 girls) imaged for sensorineural hearing loss between December 2015 and August 2017. Although most of the control patients manifested isolated hearing loss, other relevant diagnoses in this cohort included the following: Usher syndrome $(n=2)$, meningitis $(n=2)$, Waardenburg syndrome $(n=1)$, congenital cytomegalovirus infection $(n=1)$, chronic otitis media $(n=1)$, and auditory neuropathy spectrum disorder $(n=1)$.

\section{Imaging Findings}

Imaging findings in patients with branchio-oto-renal syndrome are summarized in Table 1 . The cochlea was subjectively abnormal bilaterally in all 9 patients. In 8 patients (89\%), imaging demonstrated typical unwound cochlear dysmorphology with anteromedial rotation and displacement of the middle and apical turns away from the basal turn bilaterally (Fig 2). In the remaining patient with BOR (11\%), CT demonstrated complete absence of the middle and apical turns bilaterally (Fig 3). Notably, these findings were symmetric side-to-side in all patients with BOR. By contrast, none of the control subjects demonstrated an unwound cochlea on either side. Cochlear and other inner ear abnormalities identified in control subjects are presented in Table 2. Statistically, the unwound cochlea was significantly more frequent in the BOR group compared with the control group for both reader $1(8 / 9$ BOR versus $0 / 50$ controls; $P<.001)$ and reader $2(7 / 9$ BOR versus $0 / 50$ controls; $P<.001)$. Test characteristics of the unwound cochlea for the diagnosis of branchio-oto-renal syndrome are presented in Table 3.

With regard to identification of an unwound cochlea, there was almost perfect agreement between the 2 readers $(\kappa=0.93)$, with discrepant interpretations in only 1 of $59(1.6 \%)$ cases. The single discrepant interpretation occurred in a patient with BOR with an unwound cochlea by consensus re-review (Fig 4).

The mean basal-middle turn interval was $2.5 \pm 0.13 \mathrm{~mm}$ in the 
Table 1: Summary of inner ear findings in patients with branchio-oto-renal syndrome

\begin{tabular}{|c|c|c|c|c|}
\hline $\begin{array}{l}\text { Patients } \\
\text { with BOR }\end{array}$ & $\begin{array}{l}\text { Hearing } \\
\text { Loss }\end{array}$ & $\begin{array}{l}\text { Unwound Cochlea } \\
\text { (Reader 1) }\end{array}$ & $\begin{array}{l}\text { Unwound Cochlea } \\
\text { (Reader 2) }\end{array}$ & Other Inner Ear Abnormalities \\
\hline 1 & B Mixed & Bilateral & Bilateral & $\begin{array}{l}\text { R vestibular aqueduct enlargement; B medialized facial nerve; } \\
\text { B funnel IAC }\end{array}$ \\
\hline 2 & B Mixed & Bilateral & Bilateral & $\begin{array}{l}\text { B hypoplastic apical turn/modiolus; right hypoplastic posterior } \\
\text { semicircular canal; B funnel IAC }\end{array}$ \\
\hline 3 & B Conductive & Bilateral & Bilateral & B medialized facial nerve; B funnel IAC \\
\hline 4 & B Conductive & Bilateral & Bilateral & B vestibular aqueduct enlargement; B funnel IAC \\
\hline 5 & B Conductive & Bilateral & Bilateral & $\begin{array}{l}\text { L vestibular aqueduct enlargement; B hypoplastic lateral } \\
\text { semicircular canal; L medialized facial nerve; B funnel IAC }\end{array}$ \\
\hline 6 & B Sensorineural & Bilateral & Bilateral & $\begin{array}{l}\text { B hypoplastic apical turn/modiolus; B cochlear aperture } \\
\text { stenosis; B vestibular aqueduct enlargement; B medialized } \\
\text { facial nerve; L funnel IAC }\end{array}$ \\
\hline 7 & B Sensorineural & No & No & $\begin{array}{l}\text { B cochlear hypoplasia with absent middle and apical turns; } \\
\text { B medialized facial nerve }\end{array}$ \\
\hline 8 & B Mixed & Bilateral & No & B medialized facial nerve \\
\hline 9 & B Sensorineural & Bilateral & Bilateral & $\begin{array}{l}\text { B hypoplastic apical turn/modiolus; B vestibular aqueduct } \\
\text { enlargement; B medialized facial nerve; B funnel IAC }\end{array}$ \\
\hline
\end{tabular}

Note:-IAC indicates internal auditory canal; R, right side; L, left side; B, bilateral.
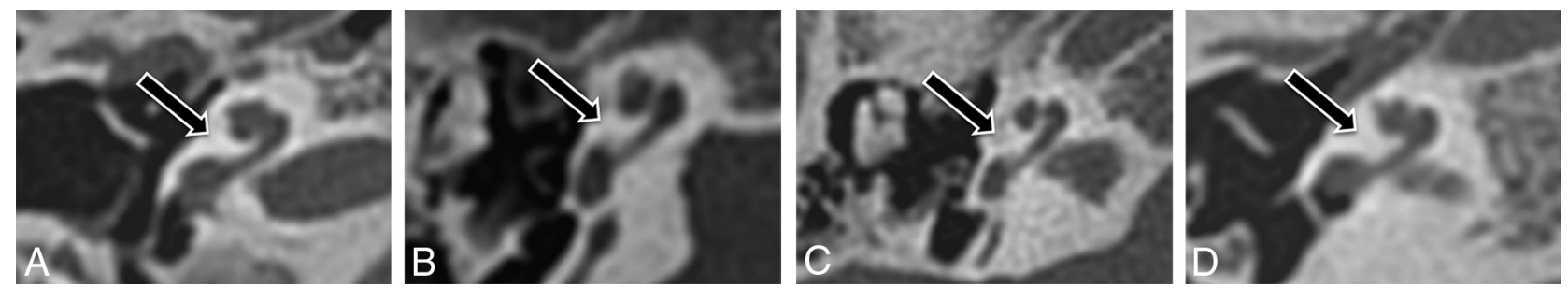

FIG 2. Cochlear morphology in patients with branchio-oto-renal syndrome. Axial CT images through the right temporal bone in 4 representative patients $(A-D)$ with unwound cochleae.

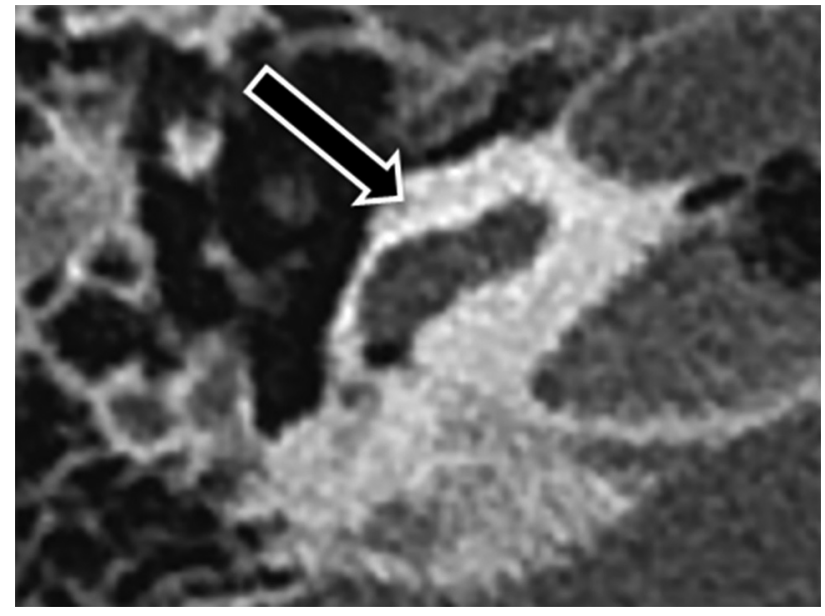

FIG 3. Branchio-oto-renal syndrome without the unwound cochlea. Axial CT image through the right temporal bone demonstrates a truncated basal turn with complete absence of the middle and apical turns of the cochlea.

BOR group compared with $1.4 \pm 0.17 \mathrm{~mm}$ in the control group. For both readers, measurements of the basal-middle turn interval were significantly larger in the BOR group compared with controls for both the right $(P<.001)$ and left $(P<.001)$ cochleae.

\section{DISCUSSION}

We report the following main findings in a cohort of children with hearing loss: The unwound cochlea is a frequent finding in patients with branchio-oto-renal syndrome; this finding was not observed in patients with hearing loss not related to BOR. There was near-perfect agreement between readers regarding the presence/absence of this subjective finding. Together, these results suggest that the unwound cochlea is a specific and reliable imaging marker of branchio-oto-renal syndrome.

Although the association between hearing loss and branchial anomalies has been recognized for more than a century, the pathogenesis of BOR remains incompletely understood. Clinical features point to a developmental defect occurring between 4 and 10 weeks of embryogenesis. ${ }^{14}$ During this interval, the inner ear develops from a pair of surface placodes that appear in human development during week 4 . These placodes fold inward to form fluid-filled sacs called vesicles; each vesicle sinks into the regional mesenchyme to form the otic capsule. The epithelium of the otic capsule then undergoes a series of morphologic changes to form the primitive membranous labyrinth, which ultimately differentiates into the cochlea and vestibular apparatus by week $8 .{ }^{10}$ During this same time, the ossicles develop from the neural crestderived cells of the first and second branchial arches, while the external auditory canal and middle ear cavity derive from the first branchial cleft and pouch, respectively; normally, elements of the branchial apparatus disappear by approximately week 8 of development. During this time, EYA1, the most commonly identified gene mutation underlying BOR, functions to promote cell survival within the developing ear. ${ }^{15}$ Interaction of EYA1 with the DNA-binding proteins SIX1 and SIX5 results in the recruitment of DNA repair (rather than proapoptotic) machinery in response to genetic damage during organogenesis. ${ }^{16}$ EYA1 has been shown to be prominently expressed by the cochlear and vestibular epithelium as well as by the mesenchyme of the otic capsule and 
Table 2: Abnormalities of the cochlea and inner ear in control patients with hearing loss not associated with branchio-oto-renal syndrome

Cochlear Abnormality

Other Inner Ear Abnormalities

Incomplete partition defect type $2(n=3)$

Cochlear aperture stenosis $(n=1)$

Labyrinthitis ossificans $(n=1)$

Table 3: Test characteristics of the unwound cochlear morphology for a clinical diagnosis of branchio-oto-renal syndrome

\begin{tabular}{lcccl}
\hline Reader & Sensitivity & Specificity & PPV & \multicolumn{1}{c}{ NPV } \\
\hline 1 & $0.89(0.51-0.99)$ & $1.0(0.91-1.0)$ & $1.0(0.60-1.0)$ & $0.98(0.88-1.0)$ \\
2 & $0.78(0.40-0.96)$ & $1.0(0.91-1.0)$ & $1.0(0.56-1.0)$ & $0.96(0.86-0.99)$
\end{tabular}

Note:-NPV indicates negative predictive value; PPV, positive predictive value. ${ }^{a}$ Numbers in parentheses are $95 \% \mathrm{Cls}$.

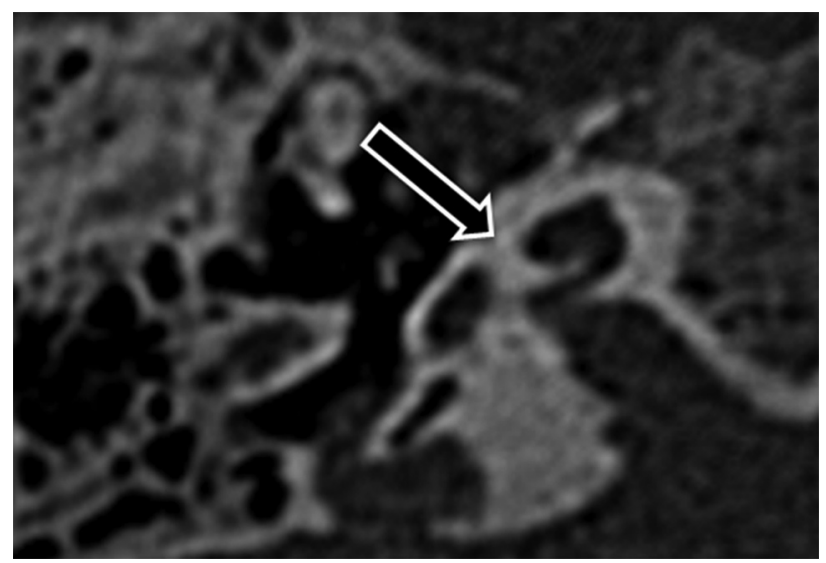

FIG 4. Interreader disagreement. Axial CT images through the right temporal bone in the single patient with BOR with interreader discrepancy. This cochlea was considered unwound at consensus re-review.

middle ear during early development. ${ }^{14}$ These findings support a direct role for EYA1 in the development of all components of the middle and inner ear, consistent with the diversity of ear findings reported in patients with BOR. ${ }^{14}$ Most interesting, EYA1 is also prominently expressed during development by the metanephric cells surrounding the ureteric branches of the kidney, consistent with a role in kidney morphogenesis. ${ }^{14}$

Branchio-oto-renal syndrome continues to present a diagnostic challenge, mainly due to its clinical and genetic heterogeneity. Given that it has become a mainstay in the evaluation of patients with hearing loss, temporal bone imaging represents an intuitively appealing opportunity to make the diagnosis. Unfortunately, studies investigating temporal bone findings at CT have yielded inconsistent results. Cochlear anomalies are the most frequently reported, typically hypoplasia of the apical turn, with a prevalence that has ranged widely across cohorts from $30 \%{ }^{17}$ to $100 \%{ }^{10,18}$ Further complicating potential application to clinical practice, the specificity of temporal bone findings has only rarely been reported. ${ }^{10}$ In response to these deficiencies, Propst et al ${ }^{10}$ compared the appearance of the temporal bones in a large cohort of patients with BOR with that of subjects with normal hearing. With regard to the cochlea, they observed isolated hypoplasia of the apical turn with a deficient modiolus in all patients with BOR, but none of the healthy controls. Consistent with their findings, we observed apical turn hypoplasia and deficiency of the modio- lus in our cohort, though only in 3 of 9 patients. Ultimately, direct comparison with Propst et al on our primary end point-the diagnostic accuracy of the unwound cochlea for BOR-is not possible because their report predates the earliest mention of the unwound coearliest mention of the

chlea that we could find in the imaging literature.

Other frequently reported inner ear anomalies in BOR include an abnormal course of the facial nerve, a dysmorphic internal auditory canal, dysplasia of the lateral and/or posterior semicircular canals, vestibular aqueduct enlargement, and cochlear aperture stenosis. ${ }^{10,17-19}$ These imaging findings, however, have been reported in other syndromes as well as in isolated forms of hearing loss. ${ }^{3,17}$ Most interesting, Propst et al ${ }^{10}$ observed a characteristic medialized course of the facial nerve (medial to the cochlea) in most patients with BOR but in none of the healthy controls. Consistent with their results, we observed a medialized facial nerve in 7 of 9 patients with BOR but in none of the patients with other causes of hearing loss. The combination of this finding with the unwound cochlea would have resulted in a sensitivity and specificity of $100 \%$ in our cohort. This observation raises the possibility that the optimal diagnosis of BOR on temporal bone CT would be based on a combination of findings, rather than a single imaging marker. Finally, our results are consistent with the original assertion by Robson ${ }^{3}$ that unwound cochlear morphology is characteristic of BOR. Our study adds to the literature by measuring the diagnostic accuracy of this finding in a cohort of children with hearing loss.

This study has several limitations. First, this was a small cohort of patients with BOR. However, the statistical power for the specificity of this finding was driven by the number of control patients, and we observed a relatively narrow confidence interval for specificity $(91 \%-100 \%)$. Still, a study with a larger cohort would be of value, especially with regard to defining the optimal combination of findings to identify these patients within the overall population of children with hearing loss. Second, genetic testing was not performed. It therefore cannot be assumed that this cohort captures the spectrum of mutations associated with BOR. This feature is particularly notable, given the observation that genotype may be related to the prevalence of inner ear anomalies. ${ }^{16}$ Third, our results reflect cochlear findings on temporal bone $\mathrm{CT}$, which is the standard test performed for hearing loss at our institution. However, MR imaging has become increasingly popular in this clinical setting, especially in light of the risks of ionizing radiation in children. Application of this finding to MR imaging will require further study. Finally, this study was not designed to evaluate the unwound cochlea as a potential causative factor in hearing loss. In fact, this finding was identified in patients without a detectable sensorineural component of hearing loss at formal audiologic evaluation. The unwound cochlear morphology may therefore reflect anomalous development of the inner ear in these patients, which is independent, at least in some cases, of the features that determine sensorineural hearing loss.

\section{CONCLUSIONS}

We report, in a cohort of children with hearing loss, that the unwound cochlea, characterized by anteromedial rotation and 
displacement of the middle and apical turns away from the basal turn, was a specific and reliable imaging marker of branchio-otorenal syndrome. These findings further support the diagnostic accuracy and therefore the utility of temporal bone imaging in the diagnosis of this challenging disorder.

\section{REFERENCES}

1. Ginat DT, Ferro L, Gluth MB. Anatomic and quantitative temporal bone CT for preoperative assessment of branchio-oto-renal syndrome. Clin Neuroradiol 2016;26:481-83 CrossRef Medline

2. Lindau TA, Cardoso AC, Rossi NF, et al. Anatomical changes and audiological profile in branchio-oto-renal syndrome: a literature review. Int Arch Otorhinolaryngol 2014;18:68-76 CrossRef Medline

3. Robson CD. Congenital hearing impairment. Pediatr Radiol 2006; 36:309-24 CrossRef Medline

4. Huang BY, Zdanski C, Castillo M. Pediatric sensorineural hearing loss, Part 2: syndromic and acquired causes. AJNR Am J Neuroradiol 2012;33:399-406 CrossRef Medline

5. Huang BY, Zdanski C, Castillo M. Pediatric sensorineural hearing loss, Part 1: practical aspects for neuroradiologists. AJNR Am J Neuroradiol 2012;33:211-17 CrossRef Medline

6. Bellini C, Piaggio G, Massocco D, et al. Branchio-oto-renal syndrome: a report on nine family groups. Am J Kidney Dis 2001;37:505-09 CrossRef Medline

7. Morisada N, Nozu K, Iijima K. Branchio-oto-renal syndrome: comprehensive review based on nationwide surveillance in Japan. Pediatr Int 2014;56:309-14 CrossRef Medline

8. Chang EH, Menezes M, Meyer NC, et al. Branchio-oto-renal syndrome: the mutation spectrum in EYA1 and its phenotypic consequences. Hum Mutat 2004;23:582-89 CrossRef Medline

9. Song MH, Kwon TJ, Kim HR, et al. Mutational analysis of EYA1, SIX1 and SIX5 genes and strategies for management of hearing loss in patients with BOR/BO syndrome. PLoS One 2013;8:e67236 CrossRef Medline
10. Propst EJ, Blaser S, Gordon KA, et al. Temporal bone findings on computed tomography imaging in branchio-oto-renal syndrome. Laryngoscope 2005;115:1855-62 CrossRef Medline

11. Chen A, Francis M, Ni L, et al. Phenotypic manifestations of branchio-oto-renal syndrome. Am J Med Genet 1995;58:365-70 CrossRef Medline

12. Fraser FC, Sproule JR, Halal F. Frequency of the branchio-oto-renal (BOR) syndrome in children with profound hearing loss. Am J Med Genet 1980;7:341-49 CrossRef Medline

13. Cohen JF, Korevaar DA, Altman DG, et al. STARD 2015 guidelines for reporting diagnostic accuracy studies: explanation and elaboration. BMJ Open 2016;6:e12799 CrossRef Medline

14. Abdelhak S, Kalatzis V, Heilig R, et al. A human homologue of the Drosophila eyes absent gene underlies branchio-oto-renal (BOR) syndrome and identifies a novel gene family. Nat Genet 1997;15: 157-64 CrossRef Medline

15. Cook PJ, Ju BG, Telese F, et al. Tyrosine dephosphorylation of H2AX modulates apoptosis and survival decisions. Nature 2009;458: 591-96 CrossRef Medline

16. Sanggaard KM, Rendtorff ND, Kjaer KW, et al. Branchio-oto-renal syndrome: detection of EYA1 and SIX1 mutations in five out of six Danish families by combining linkage, MLPA and sequencing analyses. Eur J Hum Genet 2007;15:1121-31 CrossRef Medline

17. Kemperman $\mathrm{MH}$, Koch $\mathrm{SM}$, Joosten $\mathrm{FB}$, et al. Inner ear anomalies are frequent but nonobligatory features of the branchio-oto-renal syndrome. Arch Otolaryngol Head Neck Surg 2002;128:1033-38 CrossRef Medline

18. Ceruti S, Stinckens C, Cremers CW, et al. Temporal bone anomalies in the branchio-oto-renal syndrome: detailed computed tomographic and magnetic resonance imaging findings. Otol Neurotol 2002;23:200-07 CrossRef Medline

19. Ostri B, Johnsen T, Bergmann I. Temporal bone findings in a family with branchio-oto-renal syndrome (BOR). Clin Otolaryngol Allied Sci 1991;16:163-67 Medline 